\title{
Simultaneous laparoscopic nephroureterectomy and robot- assisted anterior pelvic exenteration with intracorporeal ileal conduit urinary diversion: step-by-step video-illustrated technique
}

Éder Silveira Brazão Júnior ${ }^{1}$, Daniel Gomes Coser ${ }^{1}$, Rafael Ribeiro Meduna ${ }^{1}$, Walter Henriques da Costa ${ }^{1}$, Stênio de Cássio Zequi ${ }^{1}$

${ }^{1}$ Departamento de Urologia, AC Carmargo Cancer Center, São Paulo, SP, Brasil

\begin{abstract}
Introduction: One of the most remarkable characteristics of urothelial carcinomas is multifocality. However, occurrence of synchronous bladder cancer and upper urinary tract urothelial cancer (UTUC) is exceptional. Minimally invasive approach for these synchronous tumors was just occasionally reported (1-4). The aim of this video article is to describe step-bystep the technique for simultaneous laparoscopic nephroureterectomy and robot-assisted anterior pelvic exenteration with intracorporeal ileal conduit urinary diversion (ICUD). Patients and methods: A 66-year-old female presented with synchronous BCG refractory non-muscle invasive bladder cancer and a right-side UTUC. She was a former smoker and had previously been submitted to multiple transurethral resections of bladder tumor, BCG and right distal ureterectomy with ureteral reimplant. We performed a simultaneous laparoscopic right nephroureterectomy and robot-assisted anterior pelvic exenteration with totally intracorporeal ICUD. Combination of robot-assisted and pure laparoscopic approaches was proposed focusing on optimization of total operative time (TOT).

Results: Surgery was uneventful. TOT was of 330 minutes. Operative time for nephroureterectomy, anterior pelvic exenteration and ICUD were 48, 135, 87 minutes, respectively. Estimated blood loss was 150mL. Postoperative course was unremarkable and patient was discharged after 7 days.

Histopathological evaluation showed a pT1 high grade urothelial carcinoma plus carcinoma in situ both in proximal right ureter and bladder, with negative margins. Twelve lymph nodes were excised, all of them negative.

Conclusion: In our preliminary experience, totally minimally invasive simultaneous nephroureterectomy and cystectomy with intracorporeal ICUD is feasible. Pure laparoscopic approach to upper urinary tract may be a useful tactic to reduce total operative time.
\end{abstract}

\section{ABBREVIATIONS}

ICUD = Ileal Conduit Urinary Diversion

UTUC = Upper urinary Tract Urothelial Carcinoma

TOT $=$ Total Operative Time

\section{ACKNOWLEDGEMENTS}

\section{Statement of ethics}

Research was conducted ethically in accordance with Declaration of Helsinki. Informed consent was obtained from the patient. 


\section{CONFLICT OF INTEREST}

None declared.

\section{REFERENCES}

1. Berglund RK, Matin SF, Desai M, Kaouk J, Gill IS. Laparoscopic radical cystoprostatectomy with bilateral nephroureterectomy: initial report. BJU Int. 2006; 97:37-41.

2. Barros R, Frota R, Stein RJ, Turna B, Gill IS, Desai MM. Simultaneous laparoscopic nephroureterectomy and cystectomy: a preliminary report. Int Braz J Urol. 2008; 34:413-21.
3. Ou YC, Yang CR, Yang CK, Cheng CL, Hemal AK. Simultaneous robot-assisted nephroureterectomy and cystectomy in patients with uremia and multifocal urothelial carcinoma. J Endourol. 2011; 25:979-84.

4. Buse S, Hach CE, Alexandrov A, Mager R, Haferkamp A. Simultaneous en-bloc robot-assisted radical cystectomy and nephro-ureterectomy: technique description, outcomes, and literature summary. J Robot Surg. 2016; 10:315-322.

Submitted for publication:

November 19, 2020

Accepted after revision:

January 03, 2021

Published as Ahead of Print:

January 20, 2021

Correspondence address:

Éder Silveira Brazão Júnior, MD

Departamento de Urologia, AC Carmargo Cancer Center Rua Antônio Prudente, 211, Liberdade São Paulo, SP, 01509-010, Brasil

Telephone: + 5511945459561

E-mail: eder.brazao@accamargo.org.br

\section{ARTICLE INFO}

\section{Éder Silveira Brazão Júnior}

http://orcid.org/0000-0002-8547-2757

Available at: http://www.intbrazjurol.com.br/video-section/20201006_Brazao_et_al

Int Braz J Urol. 2021; 47 (Video \#17): 1072-3 